\title{
An Upgraded Transverse Electromagnetic Parallel Plates for Dielectric Measurement
}

\author{
S. K. Yee ${ }^{\star 1}$, R. Padu ${ }^{2}$, C. K. $\mathrm{Sia}^{3}$, X. T. I. Ngu ${ }^{4}$, S. H. Dahlan ${ }^{5}$, M. A. Azlan ${ }^{6}$ \\ ${ }^{1,2,4,5}$ Center for Applied Electromagnetic, Faculty of Electrical and Electronic Engineering, Universiti Tun \\ Hussein Onn Malaysia \\ ${ }^{3,6}$ Faculty of Mechanical and Manufacturing Engineering, Universiti Tun Hussein Onn Malaysia \\ ${ }^{\star}$ Corresponding author, e-mail: skyee@uthm.edu.my
}

\begin{abstract}
A new version of transverse electromagnetic parallel plates with irregular plates' width and plate separation has been developed for dielectric measurement. The separations between the plates are supported by four rectangular Teflon block and $1 \mathrm{~mm}$ of groove is proposed at the center of the upper plate to maintain the measurement repeatability. The groove enables the samples which are slightly higher than $2 \mathrm{~cm}$ to be fitted well between the plates without introducing extra force to the plates. The performance of both parallel plates has been compared in the frequency range from $100 \mathrm{MHz}$ to $1.1 \mathrm{GHz}$. It is found that the upgraded parallel plate offers better return loss and insertion loss above $500 \mathrm{MHz}$ compared to the previous parallel plate. It is reported from this work that the return loss of the parallel plate must be lower than $-15 \mathrm{~dB}$ in order to achieve accurate dielectric constant. However, the insertion loss of the parallel plates does not influence the real permeability significantly. The upgraded TEM parallel plate produces a consistent reading with a standard deviation of less than 0.05 above frequency $200 \mathrm{MHz}$. The dielectric measurement of Polypropylene (PP) has proven the capability of this upgraded TEM parallel plate.
\end{abstract}

Keywords: dielectric measurement, transverse electromagnetic parallel plates, dielectric constant

Copyright $@ 2017$ Universitas Ahmad Dahlan. All rights reserved.

\section{Introduction}

Dielectric measurement discloses the relative permittivity and relative permeability of a material. These parameters are very important in microwave device design, shielding and absorbing material invention. Besides that, it is also significant in food science [1], agriculture [2], Geotechnical study [3], and cancer detection [4]. These researchers are establishing the correlation between the parameter of their interest with the dielectric properties through the dielectric measurement. Since the material under test can exist in many forms such as powder, liquid, semi liquid, thin film, solid, hence the design of the dielectric sample holder to accommodate these samples become a challenging issue. Techniques and methodologies for material characterization are well established and documented, these techniques including the transmission line method [5-6] (coaxial line or waveguide is used to hold the sample), resonator [7], resonant perturbation methods [8] and free space technique [9], open-ended coaxial line technique [10]. Each of these methods has their advantages and disadvantages. The techniques to be chosen is dependent on the frequency of interest, the size of the sample, form of the samples (solid, powder, or liquid), required measurement accuracy, destructive or non-destructive testing [11].

A TEM parallel plate has been designed previously [12] as shown in Figure 1. This TEM parallel plate offers convenience in sample preparation compared to coaxial line transmission line method because the sample is in the cuboid shape. Other than this, the operating frequency of this TEM parallel plate is more extensive compared to waveguide transmission line method. During the measurement, the TEM parallel plate is connected to network analyzer and the material under test (MUT) is placed at the center of the parallel plate. Nevertheless, it is found that the current TEM parallel plate design cannot guarantee the repeatability of the measurement outcomes. This is because the sample is placed based on the intuition of the users. From the aspect of handling, two cylindrical Teflon are used to maintain the plates separation at the corner. This design is not adequate and it fails to provide plates stability

Received May 11, 2017; Revised July13, 2017; Accepted August 7, 2017 
especially when a slightly higher sample is inserted in between two plates. Not only this, when a slightly higher MUT is inserted in between the plates, additional force is exerted on both plates and it affects the contact of the taper with the inner conductor of the $\mathrm{N}$-type connector. Due to this problem, several $\mathrm{N}$-type connector has been disposed due to the enlargement of inner conductor as shown in Figure 1 (b). This trouble can be pondered from the measurement result in [12] where spike appears in between $100 \mathrm{MHz}$ to $200 \mathrm{MHz}$. Aside from this, the plates separation issue also affects the impedance of the plates along its length. This introduce more return loss to the structure and ultimately affect the dielectric measurement of the MUT.

In order to solve these problems, a newer TEM parallel plates is proposed. The newer parallel plates offer extended operating bandwidth, better repeatability and it works out the handling issue too. This new design will be further explained in the following sections. Its performance and capability will be compared and discussed in the coming sections too.
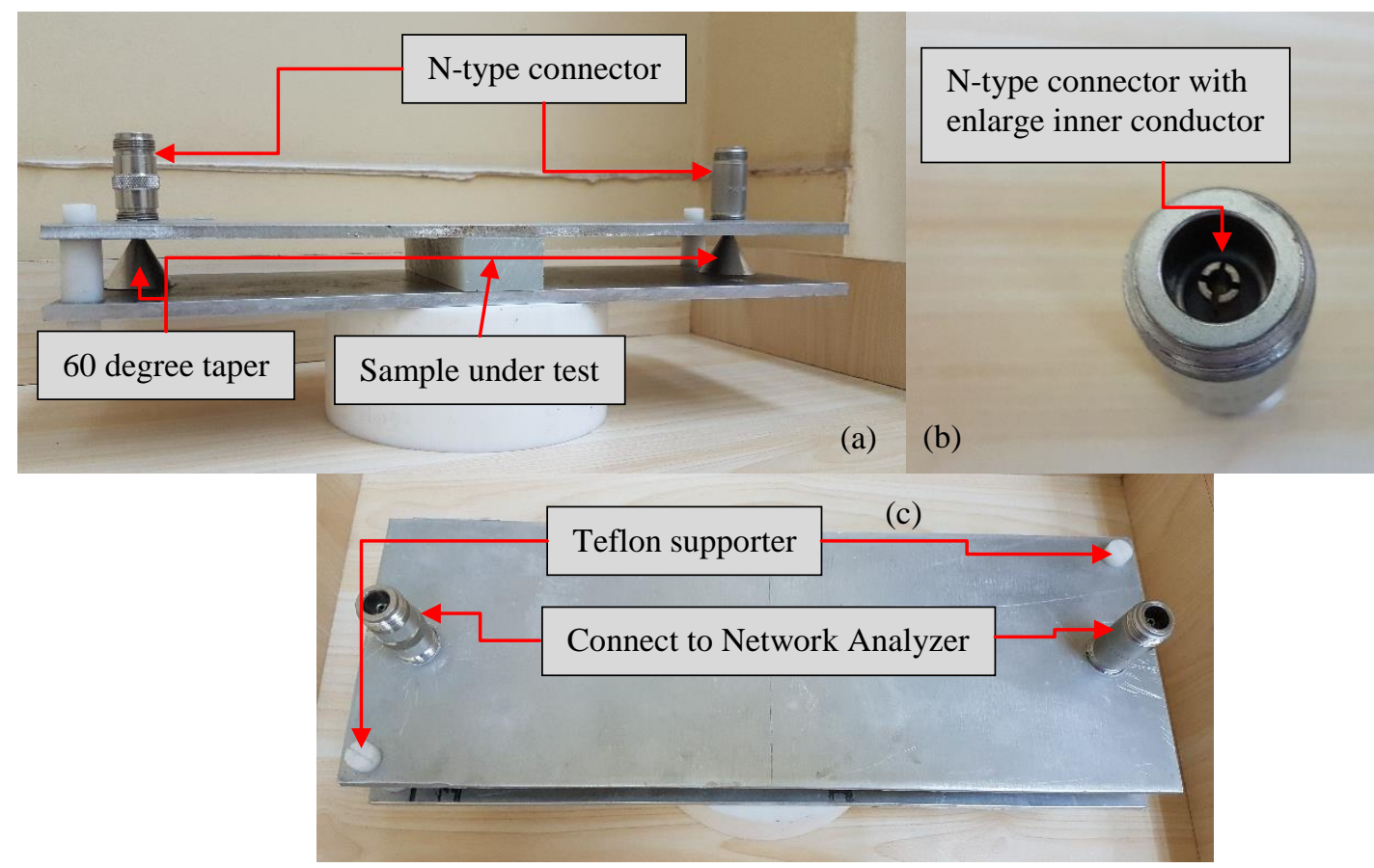

Figure 1. (a) The existing TEM parallel plate when the MUT is inserted at the center of the plate (b) Enlargement of inner conductor of $\mathrm{N}$-type connector (c) Top view of the parallel plate

\section{The Upgraded TEM Parallel Plates Design}

The upgraded TEM parallel plate is presented in Fig 2. The width of the plate has been tapered so that the operating frequency of the parallel plates can be extended. The relationships between the cutoff frequency of transverse magnetic (TM) or transverse electric (TE), with TEM modes, and characteristic impedance of the parallel plates is shown in (1) and (2). $n$ represent the mode number, $d$ is the plates separation, $w$ stands for the width of the plate, $\eta$ is the characteristic impedance of the material in between the plates, $\varepsilon$ and $\mu$ are the permittivity and permeability of the material between the plates respectively. When these equations are linked to each other as depicted in (3), it is found that the cutoff frequency is inversely proportional to the width of the plates. In order to maintain the TEM mode operation in the parallel plate, and at the same time broaden the operating frequency, the width of the place must be reduced [13].

$$
f_{c}=\frac{n}{2 d \sqrt{\varepsilon \mu}}
$$




$$
\begin{aligned}
& Z=\frac{\eta d}{w} \\
& f_{c}=\frac{n \eta}{2 Z w \sqrt{\varepsilon \mu}}
\end{aligned}
$$
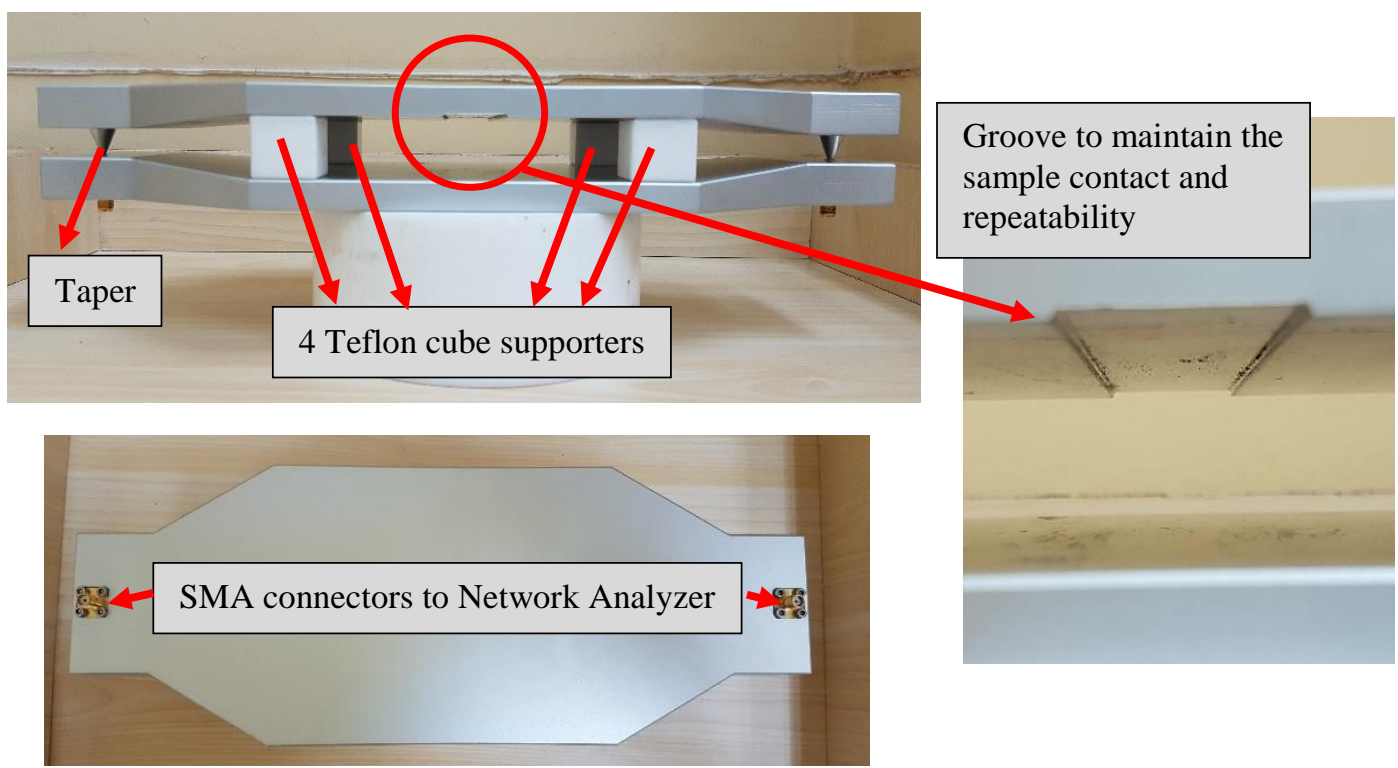

Figure 2. The design of the upgraded TEM parallel plates

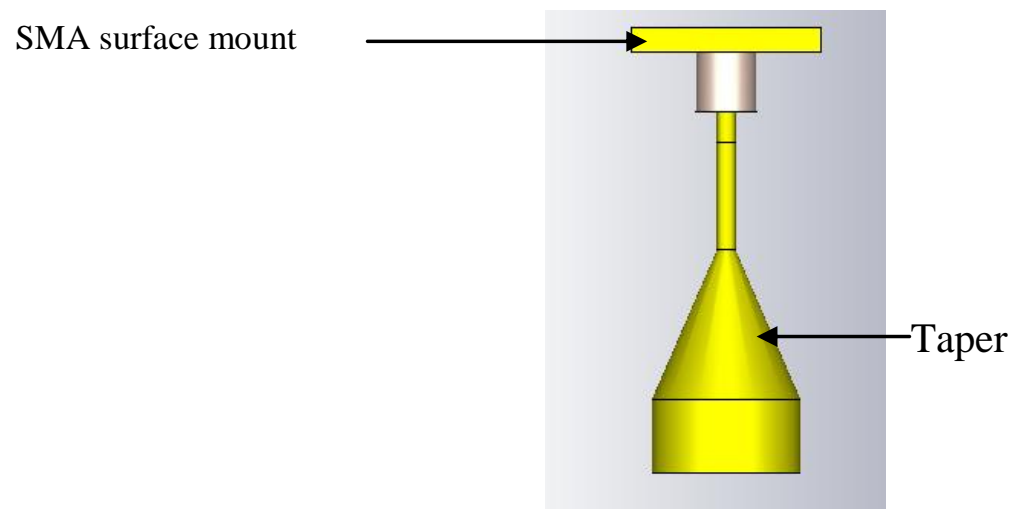

Figure 3. The connection between the SMA surface mount with the taper

In this design, the characteristic impedance of the parallel plate is very important to maintain its return loss and insertion loss. However, based on the physical dimension of the parallel plates, it is impossible to link it directly to the existing instrument in the market such as network analyzer. Hence the transition from the conventional microwave ports, such as APC 7, $\mathrm{N}$-type and SMA in the network analyzer to the parallel plate is a challenging task. The idea of tapered transmission line in impedance matching has been researched since 1981 [14], closedform equations have been shown to give the complex impedance transformed through a non- 
uniform transmission line by using exponential cosine-squared, and parabolic taper. Nevertheless, there is no clear relationship yet that describe the impedance matching transition for the parallel plates, hence in this work the idea of the taper is used and its angle and location are optimized based on CST MICROWAVE STUIDOB.

In this new design, female SMA plug and square flange has been proposed to replace the N-type connector. The inner conductor of this SMA plug is connected to the taper to avoid the problem as in the previous design. Figure 3 illustrates the connection between the taper and SMA plug in the simulation software. A pair of taper is placed at the ends of the parallel plates to provide matching in between the parallel plates and the conventional microwave port.

Previously, the users placed their sample at the center of the parallel plates based on their instinct. These often introduce uncertainty when the measurement is repeated. Hence a groove is recommended at the top plate as in Figure 2. Solid MUT can be placed at the same location when the measurement is repeated. Besides that, the other benefit of the groove is it can accommodate slightly higher solid MUT. Based on the previous experience, it is very hard to prepare a MUT with precise height. When the height of the MUT is exactly $2 \mathrm{~cm}$, it is real difficult to insert it in between the plates. However, if the height of the MUT is less than $2 \mathrm{~cm}$, it introduces an air gap that brings down the measurement accuracy. When the groove is introduced to the parallel plate, its $1 \mathrm{~mm}$ depth provides more tolerance for solid MUT that has a height of $2 \mathrm{~cm}$. The dimension of the groove is $2 \mathrm{~cm}$ width and $1 \mathrm{~mm}$ depth. Based on the simulation, it is concluded that the groove will not affect the performance of the parallel plate. Four cubes of Teflon have been located close to the midpoint of the parallel plates as the supports to hold the plate separation, the dimension and location of these Teflon blocks are optimized by using CST MICROWAVE STUIDO®.

\section{Dielectric Measurement of Polypropylene (PP)}

In order to evaluate the performance of this newly upgraded TEM parallel plates, it is used to measure the dielectric properties of PP which its properties has been published in [15]. The dielectric measurement setup has been performed as indicated in Figure 4. In this setup, the upgraded TEM parallel plates is connected to the network analyzer (NA) and the MUT is inserted in the middle of the plates. The MUT is placed in the groove section for the sake of repeatability. Although the groove design is mean for the sample width of $2 \mathrm{~cm}$, this upgraded TEM parallel plates can accommodate wider sample too as long as the sample width is less than the wavelength of electromagnetic wave in the material, $\lambda_{m}[15]$.

In this study, the Nicolson-Ross-Weir (NRW) conversion formulation will be employed to convert the $S_{11}$ and $S_{21}$ to the dielectric properties of material [16]. Even so this conversion formulation is applicable only when the calibration plane is up to the surface of the MUT. In this setup, since the calibration plane is not on the surface of the MUT, hence (4) and (5) must be applied first before the substitution to NRW conversion formulation. $S_{11}$ and $S_{21}$ are the return loss and insertion loss that is ready for the NRW formulation. The superscript $M$ and $E$ represent the s-parameter with MUT inserted and without MUT inserted respectively, $\beta$ is the phase constant and the $d$ is the thickness of the sample. [17]

$$
\begin{aligned}
& S_{11}=\frac{S_{11}^{M}-S_{11}^{E}}{S_{21}^{E} \times e^{j \beta d}} \\
& S_{21}=\frac{S_{21}^{M}}{S_{21}^{E}}
\end{aligned}
$$



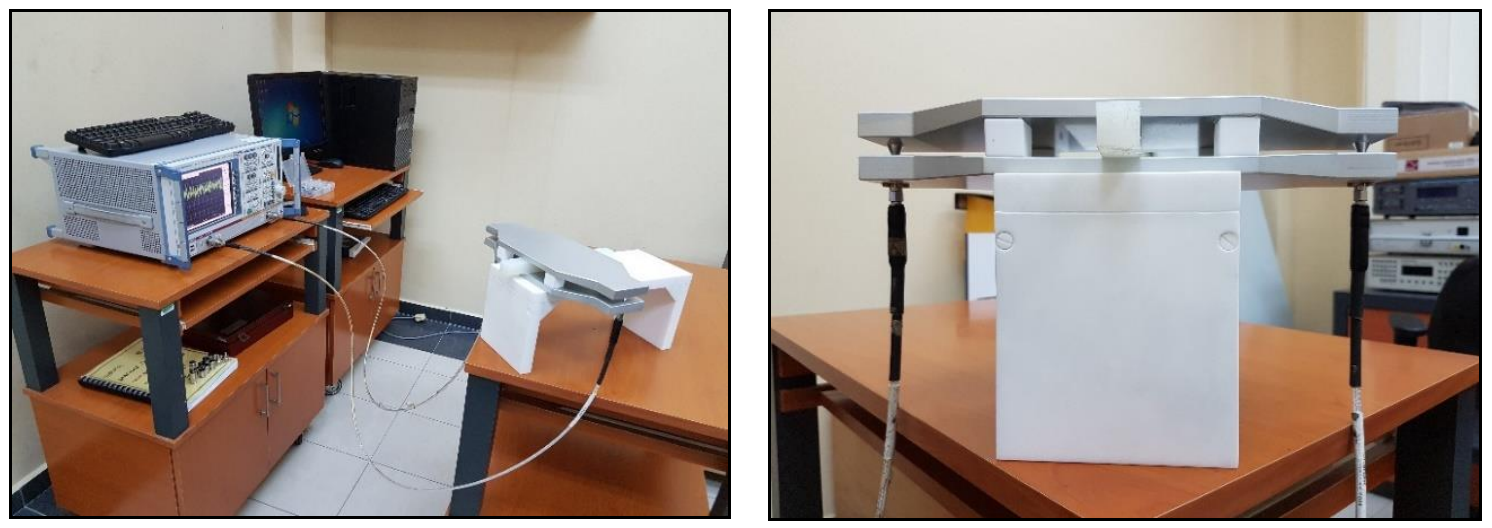

Figure 4. The dielectric measurement setup of upgraded TEM parallel plates

\section{Results and Analysis}

In this section, the return loss and insertion loss comparison of both parallel plates will be presented. Based on the comparison, it is found that they are closely related to the converted dielectric properties of PP. The dielectric measurement is repeated for five times and the results of measurement strongly support repeatability of the upgraded parallel plates.

\subsection{Comparison of Return Loss and Insertion Loss}

The dielectric measurement reveal the properties of material based on the insertion loss $\left(S_{21}\right)$ and return loss $\left(S_{11}\right)$ of EM wave through the MUT. A good specimen holder should provide the lowest (in unit decibels $(\mathrm{dB})$ ) return loss and highest (in unit $\mathrm{dB}$ ) insertion loss, so that the measured reflection and transmission is due to the MUT. Figure 5(a) and 5(b) illustrated the comparison of $S_{11}$ and $S_{21}$ of both TEM parallel plates in $\mathrm{dB}$. The previous TEM parallel plates is operating from $50 \mathrm{MHz}$ to $800 \mathrm{MHz}$ whereas the upgraded TEM parallel plates can operate up to $1100 \mathrm{MHz}$.

It is found that the upgraded TEM parallel plate offer similar $S_{11}$ (below $-20 \mathrm{~dB}$ ) in between $100 \mathrm{MHz}$ to $450 \mathrm{MHz}$ as the previous TEM parallel plates. Yet as the operating frequency increases, the reflection from the TEM parallel plate become higher in the previous design. However, the upgraded TEM parallel plates manage to offer lower $S_{11}$ (in between $30 \mathrm{~dB}$ to $-20 \mathrm{~dB}$ ) in the frequency range of $500 \mathrm{MHz}$ to $700 \mathrm{MHz}$. The $S_{11}$ fluctuates around $20 \mathrm{~dB}$ up to $1100 \mathrm{MHz}$.

On the other hand, it is found that the previous TEM parallel plates has $S_{21}$ ranging from $-0.2 \mathrm{~dB}$ to $-3.6 \mathrm{~dB}$ from $50 \mathrm{MHz}$ to $800 \mathrm{MHz}$ whereas the upgraded TEM parallel plates contribute higher insertion loss of $-0.15 \mathrm{~dB}$ to $-1.7 \mathrm{~dB}$. Based on the comparison in fig. 6 (b), it is concluded that the upgraded TEM parallel plates has better return loss and insertion loss compare to the previous TEM parallel plate.

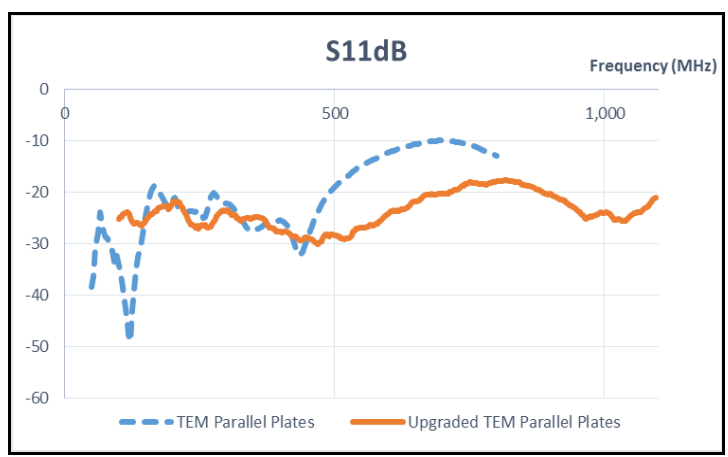

(a)

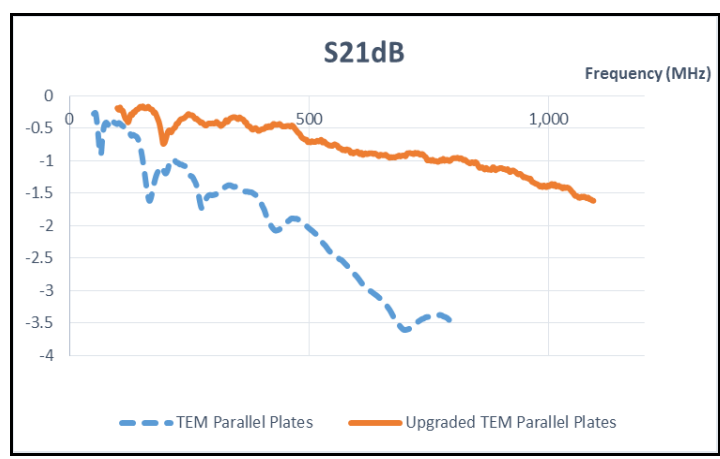

(b)

Figure 5. The return loss $\left(\mathrm{S}_{11} \mathrm{~dB}\right)$ and insertion loss $\left(\mathrm{S}_{21} \mathrm{~dB}\right)$ comparison of both parallel plates 


\subsection{Comparison of Dielectric Propertis of PP from Both Parallel Plate}

Figure 6 illustrates the comparison of dielectric constant and relative permeability of PP by using two different TEM parallel plate. It is noted that both parallel plate present similar dielectric results for PP, which is 1.9 and 1 for dielectric constant and real permeability respectively. This reading is quite close to the reading presented in [15]. It is observed from Figure 6(a) that the dielectric constant and real permeability obtained from the upgraded TEM parallel plates is more uniform and consistent throughout the whole frequency range compare to the previous parallel plates. The results from the previous TEM parallel plates start to diverge from the uniformity above $600 \mathrm{MHz}$. This can also be observed from the real permeability result in Figure 6(b). By comparing this scenario with the return loss of the parallel plate in Figure 5(a), it is found that its return loss has become higher than $-15 \mathrm{~dB}$ at frequency above $600 \mathrm{MHz}$. Other than this, the effect of the return loss to the dielectric constant of the measurement can also be reflected from the small fluctuation at $800 \mathrm{MHz}$ in the case of upgraded parallel plate. The slight increase of return loss alters the uniformity of the dielectric constant results at the same frequency range (refer Figure 6(a)). However, the performance of the return loss does not influence the real permeability much. Since PP is not a magnetic material, it should have unity of permeability as depicted in Figure $6(\mathrm{~b})$. Hence, it is concluded that the dielectric constant is more sensitive to the return loss performance of the parallel plate compared to its insertion loss. The return loss should be less than $-15 \mathrm{~dB}$ to achieve acceptable dielectric results.

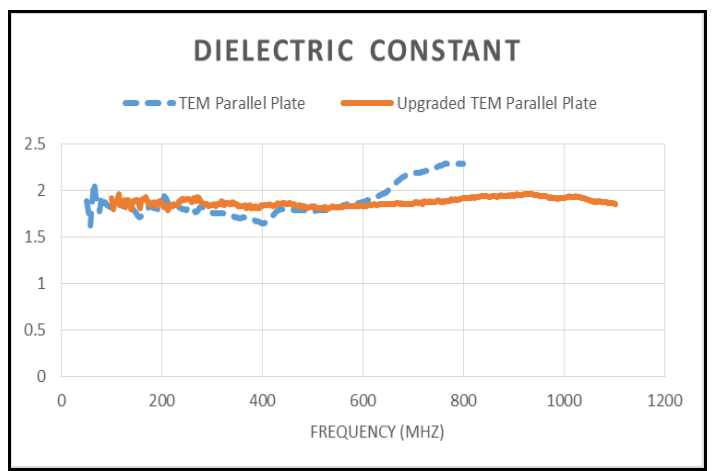

(a)

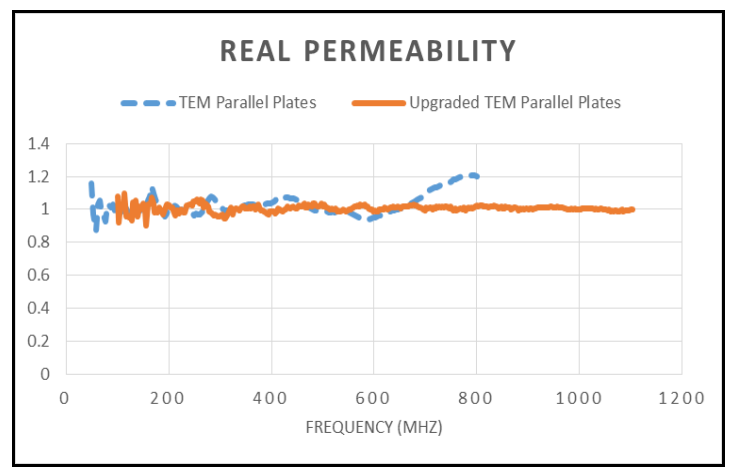

(b)

Figure 6. The comparison of dielectric properties of PP

\subsection{The Repeatability Of The Dielectric Measurement By Using The Upgraded TEM Parallel Plate}

A measuring device should be able to produce consistent reading when the measurement is repeated. In the previous parallel plate design, the users locate the MUT based on their assumption that it is at the center of the plate, this creates a great uncertainty that affect the repeatability of the measurement. In this new design, a groove is introduced to resolve this problem. Five times of measurement have been conducted on different day to evaluate the repeatability of the new design. The dielectric constant results on different day are presented in Figure. 7.

In general the measurement of PP can be repeated well, especially at frequencies above $200 \mathrm{MHz}$. The difference is larger at lower frequencies especially for the measurement repeated on day 2. This is suspected to be caused by the random error from the experimentor as the other measurements repeated at other day are matching well. The standard deviation of the measurement is illustrated in Figure 8. At frequency below $200 \mathrm{MHz}$, the standard deviation of the measurement is very high as expected due to the measurement at day 2 . However, the standard deviation is below 0.05 for the frequency above $200 \mathrm{MHz}$. This has tested the upgraded TEM parallel plate is reliable in performing the dielectric measurement and the reading is repeatable. 


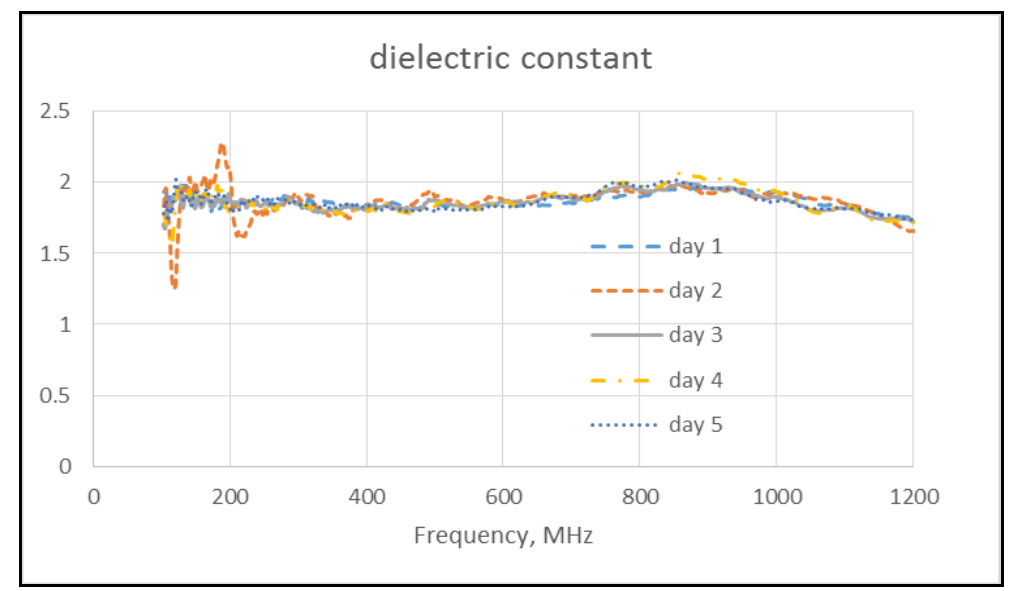

Figure 7. The dielectric constant results based on upgraded TEM parallel plate for five days

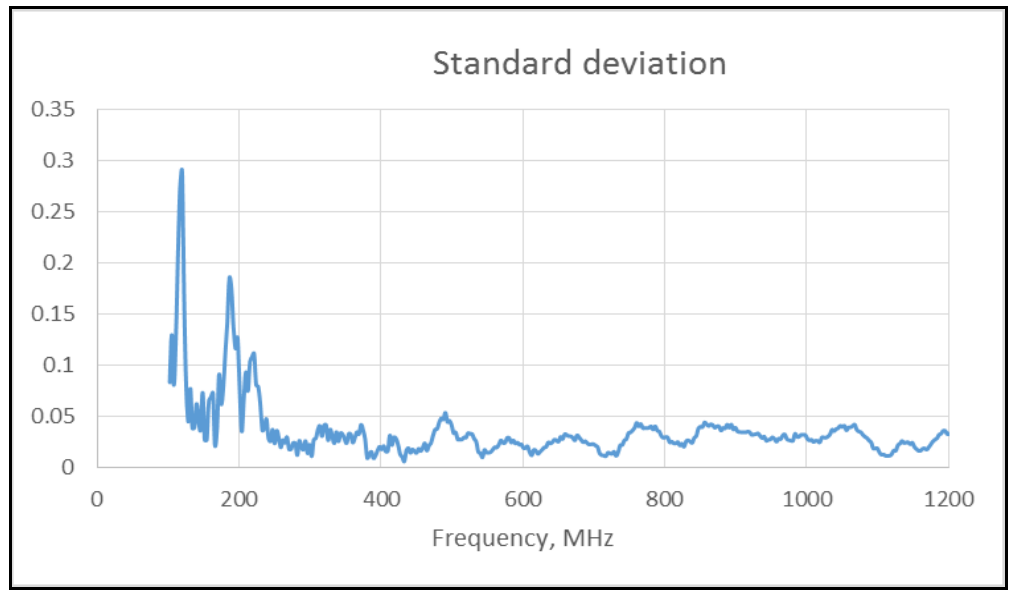

Figure 8. The standard deviation of the measurement

\section{Conclusion}

In this work a upgraded TEM parallel plate has been presented. The new design has successfully solve the connection problem of the parallel plate to the conventional microwave port by using the SMA surface mount panel. This newer design has been fabricated based on computer numerical control (CNC) technology and hence precise dimensions are achieved. The operating frequency of the parallel plate has been upgraded to $1.1 \mathrm{GHz}$ by tapering the width and distance of the parallel plate. The groove propose in this design has improved the repeatability of the measurement by introducing 0.05 of standard deviation. The dielectric measurement of PP has proven the competency of this upgraded parallel plates. Based on the results comparison, it is found that the return loss has close relationship with the dielectric constant. It is proposed that the TEM parallel plate should have return loss lower than $-15 \mathrm{~dB}$ in order to achieve consistent and reliable results.

\section{Acknowledgements}

The authors would like to thank the Office Of Research Innovation Commercialization And Consultancy Management (ORICC) fund, Vot No E15501, Universiti Tun Hussein Onn Malaysia for supporting the expenses to this conference. 


\section{References}

[1] C LIU, L. Zhang, J Peng. Effect of temperature on dielectric property and microwave heating behavior of low grade Panzhihua ilmenite ore. Transactions of Nonferrous Metals Societey of China. 2013; 23(11): 3462-3469.

[2] F Chengyan, Y Zhi, C Yuqiong. Researches on the dielectric property of fresh tea leaves. Journal of Huazhong Agricultural University. 2014; 020

[3] Liu, Haorui, Heli Yang, Fengyan Yi. Experimental study of the complex resistivity and dielectric constant of chrome-contaminated soil. Journal of Applied Geophysics. 2016; 131: 109-116.

[4] Mayrovitz, Harvey N, Daniel N Weingrad, Lidice Lopez. Patterns of temporal changes in tissue dielectric constant as indices of localized skin water changes in women treated for breast cancer: a pilot study. Lymphatic research and biology. 2015; 13(1): 20-32.

[5] Piuzzi, Emanuele, et al. Measurement system for evaluating dielectric permittivity of granular materials in the 1.7-2.6-GHz band. IEEE Transactions on Instrumentation and Measurement. 2016; 65(5): 1051-1059.

[6] Kato Yuto, et al. New uncertainty analysis for permittivity measurements using the transmission/reflection method. IEEE Transactions on Instrumentation and Measurement. 2015; 64(6): 1748-1753.

[7] Shimizu, Takashi, Yoshinori Kogami. High precision measurement method for dielectric film materials by a novel $V$ band cavity resonator. Microwave Symposium (IMS). 2014; 1-3.

[8] Jha, Abhishek Kumar, Mohammad Jaleel Akhtar. An improved rectangular cavity approach for measurement of complex permeability of materials. IEEE Transactions on Instrumentation and Measurement. 2015; 64(4): 995-1003.

[9] Orlob, Christian, et al. Compact unfocused antenna setup for X-band free-space dielectric measurements based on line-network-network calibration method. IEEE Transactions on Instrumentation and Measurement. 2013; 62(7): 1982-1989.

[10] Meaney, Paul M, et al. Open-ended coaxial dielectric probe effective penetration depth determination. IEEE Transactions On Microwave Theory And Techniques. 2016; 64(3): 915-923.

[11] Note, Agilent Application. Agilent Basics of Measuring the Dielectric Properties of Materials. Agilent Literature Number (2006).

[12] Yee SK, et al. Design and calibration of a wideband TEM-cell for material characterization. Electromagnetic Compatibility (APEMC), 2012: 749-752.

[13] Pozar, David M. Microwave engineering. John Wiley \& Sons, 2009.

[14] Ahmed, M Jamil. Impedance transformation equations for exponential, cosine-sqaared, and parabolic tapered transmission lines. IEEE Transactions on Microwave Theory and Techniques. 1981; 29(1): 67-68.

[15] Anderson EW, DW McCall. The dielectric constant and loss of polypropylene. Journal of Polymer Science Part A: Polymer Chemistry. 1958; 31(122): 241-242.

[16] Ghodgaonkar DK, VV Varadan, VK. Varadan. Free-space measurement of complex permittivity and complex permeability of magnetic materials at microwave frequencies. IEEE Transactions on instrumentation and measurement. 1990; 39(2): 387-394.

[17] Yee, See Khee. Electromagnetic shielding effectiveness of cement powder mixed with graphite for mitigation against electromagnetic interference. Diss. Universiti Tun Hussein Onn Malaysia, 2015. 